\title{
Actualización \\ Muerte súbita en deportistas jóvenes
}

\begin{abstract}
Resumen
En el presente artículo se define la muerte súbita específicamente vinculada al deporte, se describe la epidemiología, la fisiopatogenia, las causas principales así como las estrategias para su prevención y los criterios para la toma de decisiones frente a poblaciones de riesgo.A pesar de ser un fenómeno infrecuente en este grupo etario, es un hecho que produce un gran impacto personal y familiar y en el cual los exámenes médicos suelen ser ineficaces para prevenir.La relación entre la práctica de actividad física y la muerte súbita puede considerarse como ambivalente, y el impacto global de la actividad física en la muerte súbita es muy pequeño.Al diseñar estrategias preventivas, la edad de la población es útil para diferenciar acciones, siendo en general los 35 años considerados para definir dos grupos bien diferenciados, con distintas causas principales de muerte súbita.Antes de los 35 predominan las patologías congénitas, mientras que en los atletas mayores la enfermedad coronaria es la causa más frecuente.La prevención de la muerte súbita asociada con el deporte debe asentarse en tres pilares fundamentales: 1) el reconocimiento médico precompetitivo, 2) la instauración de los mecanismos necesarios para una resucitación cardiopulmonar y desfibrilación, y por último, 3) la elaboración de registros nacionales donde todas las muertes queden registradas. Una anamnesis profunda, un examen físico y un electrocardiograma normales hacen que la probabilidad de muerte súbita relacionada al deporte sea muy baja.
\end{abstract}

\section{Introducción}

Se entiende por muerte súbita a aquella que ocurre de forma inesperada, por causa natural, no traumática ni violenta, y en un corto periodo de tiempo.Se considera relacionada al deporte cuando los síntomas aparecen durante o en la hora siguiente a la práctica deportiva.

La muerte súbita de una persona joven y aparentemente sana, aun siendo muy infrecuente, es un hecho que produce un gran impacto social y familiar, sobre todo cuando tiene lugar durante la práctica deportiva.

En nuestra sociedad se tiene la creencia de que las cualidades físicas de los deportistas son un reflejo de su estado de salud y a la opinión pública le cuesta comprender cómo un joven aparentemente sano puede fallecer mientras muestra una gran vitalidad en su práctica deportiva. Sin embargo, de forma esporádica e inesperada un deportista muere súbitamente durante el entrenamiento o competición.Por otro lado, los exámenes médicos suelen ser ineficaces para su prevención.

\section{Epidemiología}

La muerte súbita puede ocurrir a cualquier edad y en personas aparentemente sanas. El soldado griego Pheidippides, que falleció después de correr desde Maratón hasta Atenas en el año 490ac es considerado el primer caso de muerte súbita descripto en la literatura en relación con el deporte.

Se calcula que aproximadamente el $12,5 \%$ de las defunciones que se producen de forma natural son muertes súbitas y, de estas, el $88 \%$ son de origen cardíaco.Según un informe de la Organización Mundial de la Salud, la mortalidad por muerte cardíaca súbita en la primera hora en personas de 20 a 64 años varía, según los países, entre 19 y 159 por 100.000 en varones y entre 2 y 35 por 100.000 en mujeres. La muerte coronaria es rara entre los 25 y 44 años (menos de 2 por 100.000 ) y aumenta a partir de esa edad ${ }^{1}$. Afortunadamente, la incidencia de muerte súbita en deportistas es baja. Son pocos los registros con autopsia publicados de muerte súbita en deportistas durante la práctica o después de ella.En Argentina no existen registros de muerte súbita. Los datos publicados provienen de Europa y EE.UU. Entre los 25 millones de deportistas que existen en los EE.UU., se estima que ocurren 1 a 5 casos de muerte súbita por millón por año. La incidencia estima- da de muerte súbita es de 1/200.000/año en deportistas jóvenes y de 1/18.000/año en el grupo mayor de 35 años $^{23}$.

La edad no solo condiciona la prevalencia de muerte súbita sino también la causa del fallecimiento, teniendo este hecho gran trascendencia a la hora de diseñar algoritmos de rastreo para valorar el riesgo en grupos de poblaciones de deportistas. Aunque hay autores que colocan el límite en los 30 años, 35 parece ser la edad que separa dos grupos bien diferenciados ${ }^{45}$.

\section{Fisiopatogenia}

La patología cardiovascular es la causa mas frecuente de muerte, al igual que en la muerte súbita no asociada al deporte.En el deportista joven (menor de 35 años) predominan las patologías congénitas, mientras que en el atleta de mayor edad la enfermedad coronaria es la causa más frecuente.

Cada vez se practica más deporte, tanto en forma competitiva como recreacional.Esta situación ha condicionado que la sociedad demande a la comunidad médica información más precisa sobre las recomendaciones que ha de seguir para practicar cualquier deporte con el menor riesgo posible.

\section{Mecanismos favorecedores o desencadenantes de muerte súbita}

La relación entre la práctica de actividad física y la muerte súbita puede considerarse como ambivalente. Por una parte se ha reconocido que la práctica regular de actividad física es un factor protector de cardiopatía isquémica, y por otra, se sabe que la actividad física intensa puede desencadenar una muerte súbita, sobre todo en personas que no realizan este tipo de actividades de forma regular. El impacto global de la actividad física sobre la muerte súbita es relativamente pequeño, ya que la incidencia anual es muy baja: 1:200.000 en jóvenes.

Las personas que practican actividad deportiva intensa presentan una incidencia mayor de muerte súbita que las no deportistas:1,6 muertes por 100.000 vs. 0,75 por $100.000^{6.8}$.

Por otro lado, en un estudio publicado sobre muerte súbita en general se observó que casi el $70 \%$ de las personas que fallecieron estaban en reposo en el momento de presentarse el episodio. 
Los principales mecanismos involucrados guardan relación con los cambios hemodinámicos y electrofisiológicos que se producen durante el ejercicio que, además, pueden ser distintos según el tipo de ejercicio realizado. La actividad deportiva induce una serie de adaptaciones morfológicas y funcionales en el corazón humano directamente relacionadas con el tipo, duración e intensidad del entrenamiento, y con los años de práctica deportiva. Su expresión clínica depende de factores genéticos, metabólicos, humorales y, en gran medida, del tipo de entrenamiento. En aquellos deportes en los que predomina el ejercicio dinámico con participación de grandes masas musculares, el aporte de oxígeno a los músculos activos se realiza fundamentalmente a partir del metabolismo aeróbico y, por lo tanto, se traduce en un importante aumento del consumo de oxígeno. Esto condiciona una sobrecarga de volumen sobre el ventrículo izquierdo y, como consecuencia, normalmente se incrementa la masa cardíaca y un remodelado estructural de corazón. Esta hipertrofia fisiológica o corazón de atleta es considerado como una adaptación benigna al entrenamiento físico sistemático sin consecuencias cardiovasculares adversas.Por el contrario, los deportes con predominio del ejercicio estático o explosivo y una demanda energética fundamentalmente anaeróbica, apenas aumentan el consumo de oxígeno y origina escasas modificaciones en la morfología cardiaca ${ }^{5}$.

En el $40 \%$ de los atletas se pueden observar alteraciones electrocardiográficas (aumento del voltaje de los complejos QRS, ondas $Q$, anormalidades de la repolarización) como parte de la llamadas adaptaciones fisiológicas. Además, debido al tono vagal aumentado, los atletas entrenados sin enfermedad cardíaca estructural están también sujetos a arritmias y alteraciones de la conducción que no requieren tratamiento invasivo o específico ${ }^{9}$.

Durante el ejercicio físico se produce un aumento de las catecolaminas circulantes, que además se ve incrementado por el estrés que genera la competición y que exagera las respuestas de la presión ar terial y la frecuencia cardíaca y, por lo tanto, del consumo de oxígeno miocárdico.

Algunos fármacos pueden inducir y provocar arritmias ventriculares malignas y muerte súbita, como por ejemplo, algunos diuréticos y algunos antiarrítmicos clase IA e IC.

El consumo de cocaína tiene efectos cardiovasculares no deseados que son dependientes de la dosis y de la duración del consumo. La cocaína es un potente simpaticomimético que favorece la vasoconstricción coronaria, la isquemia miocárdica y el infarto ${ }^{10}$ También los cambios ambientales extremos y/o el estrés añadido que se genera en deportes con elevado componente emocional pueden incrementar significativamente la demanda miocárdica de oxígeno y el riesgo de muerte súbita en deportistas susceptibles ${ }^{11}$.

\section{Causas en deportistas menores de 35 años}

El riesgo de muerte súbita en este grupo de edad es excepcionalmente pequeño, pero cuando ocurre siempre es inesperado y con gran repercusión social y en los medios de comunicación.

Las patologías responsables de fallecimiento guardan también relación con la edad;así, en los deportistas jóvenes las causas son generalmente congénitas y casi nunca de origen isquémico.En las series americanas las tres principales causas de muerte en este grupo de edad son la micardiopatía hipertrófica, la secundaria a un traumatismo torácico (conmotio cordis) y las anomalías congénitas de las arterias coronarias (ver cuadro 1).Sin embargo, las estadísticas del continente europeo ofrecen resultados sensiblemente diferentes, siendo la miocardiopatía arritmogénica (MCA) y las miocarditis las patologías mas frecuentes ${ }^{12-16}$.
En 1995 se publicó la primera serie de muertes secundarias a un traumatismo no penetrante de tórax durante la práctica deportiva (conmotio cordis) mereciendo este extraño fenómeno una mención especial por que se asocia con muerte súbita en jóvenes con edad promedio de 14 años ${ }^{17}$.La misma se produce por un impacto directo no penetrante en el tórax sobre la región cardíaca, con un objeto contundente que actúa a modo de proyectil, en personas susceptibles y en un momento concreto del ciclo cardíaco:la fase vulnerable de la repolarización que comprende 15 a 30 milisegundos antes del pico de la onda T (representa el 1\% del ciclo cardiaco total $)^{18}$. En estos casos no existe una cardiopatía de base y no se encuentra una causa estructural que favorezca la muerte.

Si bien no se determinó la causa de muerte en el 30\% de los casos, en una serie española la patología predominante en los menores de 30 años fue la MCA (21.8\%). Este hallazgo corrobora que el deporte es un factor de riesgo en los pacientes con esta enfermedad. A diferencia de las series del norte de Europa, la miocarditis sólo estuvo presente en el grupo de no deportistas y las anomalías de las arterias coronarias (tercera causa de muerte en las series americanas e italianas) presentaron una escasa prevalencia.

Las diferencias en las causas de muerte pueden deberse a una particular predisposición genética o más bien a un resultado positivo de la instauración desde hace ya varias décadas en Italia, de un programa único nacional de rastreo de atletas antes de participar de competencias deportivas ${ }^{1920}$.

\section{Cuadro 1: causas de muerte súbita en atletas jóvenes}

\begin{tabular}{l|c|c|c}
\hline Causa & EE.UU. & España & Italia \\
\hline Miocardiopatia hipertrófica (MCH) & $26,4 \%$ & $6,5 \%$ & $7,3 \%$ \\
\hline Conmotio cordis & $19,9 \%$ & No hay registro & \\
\hline Anomalías de las arterias coronarias & $13,7 \%$ & $3,2 \%$ & $12,2 \%$ \\
\hline Hipertrofia ventricular izquierda sin causa aparente & $7,5 \%$ & $4,9 \%$ & $7,2 \%$ \\
\hline Miocarditis & $5,2 \%$ & $9,3 \%$ & $6,1 \%$ \\
\hline Miocardiopatía arritmogénica & $2,8 \%$ & $21,8 \%$ & $22,4 \%$ \\
\hline Indeterminadas & - & $16,3 \%$ & - \\
\hline Arteriosclerosis coronaria & - & - & $18,4 \%$
\end{tabular}

\section{Estrategias para la prevención}

La prevención de la muerte súbita asociada con el deporte debe asentarse en tres pilares fundamentales:1) el reconocimiento médico precompetitivo, 2) la instauración de los mecanismos necesarios para una resucitación cardiopulmonar y desfibrilación, y por último, 3) la elaboración de registros nacionales donde todas las muertes queden registradas.

A la hora de identificar poblaciones de riesgo para establecer estrategias de prevención deben tenerse en cuenta algunos aspectos fundamentales como la relación entre el número absoluto de eventos y la incidencia de muerte en poblaciones de riesgo definidas, que se trata de un riesgo dependiente del tiempo y la eficacia de las posibles intervenciones preventivas y terapéuticas. Por otro lado, las estrategias de prevención pueden ser de tipo poblacional o centradas únicamente en los grupos de alto riesgo. Así, si aplicamos la estrategia en la población general necesitamos hacérsela a 200.000 jóvenes para prevenir una muerte. En este contexto, los costos y los riesgos relacionados con los potenciales beneficios de las intervenciones limitan la efectividad y la eficiencia de estos programas.

El principal objetivo del reconocimiento cardiológico precompetitivo debe ser detectar de forma precoz aquellas patologías cardíacas, especialmente las silentes, capaces de constituir un riesgo de muerte súbita. Existe controversia en cuanto a los métodos 
diagnósticos necesarios, su capacidad para identificar a sujetos con riesgo y su eficacia para modificar la historia natural de las enfermedades que con mayor frecuencia se asocian a muerte súbita durante la actividad deportiva. Sin embargo, son fundamentalmente motivos económicos los que priman a la hora de implementar este tipo de reconocimientos ya que no son costoefectivos, y en algunos países como EE.UU., además no son bien aceptados debido a que se los considera una intromisión en el derecho a la intimidad y libertad de las personas. En Italia existe desde 1971 una ley de protección médica para actividades deportivas que obliga a sus deportistas a obtener un certificado de idoneidad previo a la competencia.

Para diseñar un protocolo de reconocimiento precompetitivo se deben responder algunas preguntas: ¿Qué debe buscar?; ¿Qué pruebas debe incluir?; y ¿Cuál es su eficacia?

La primera pregunta tiene una respuesta relativamente fácil, ya que entre la gran variedad de cardiopatías descriptas como responsables de muerte súbita en el deportista joven, algunas ocurren con mayor frecuencia. Así, en EE.UU. y según las observaciones en autopsias, la $\mathrm{MCH}$, el commotio cordis y las anomalías coronarias representan cerca de dos tercios de todas las causas ${ }^{12}$ mientras que en Europa, la MCA constituye un $25 \%$ del total ${ }^{71421}$.

En cuanto a las pruebas que debe incluir y su eficacia, el reconocimiento ha sido objeto de diversas especulaciones. Algunos, basados en criterios de costo-efectividad, preconizan la realización exclusiva de la valoración de los antecedentes y una exploración física, mientras que en el otro extremo se encuentra el modelo italiano, que incluye además un electrocardiograma de reposo, una prueba de esfuerzo submáxima y un ecocardiograma.

En principio el protocolo debe ser sencillo para que pueda se aplicado ampliamente, pero siempre conservando la capacidad para identificar la presencia de patología cardiaca de riesgo.

Las Asociaciones Americanas del Corazón y de Medicina del Deporte establecen, en deportistas de competición, la necesidad de realizar un cuidadoso estudio de la historia personal y familiar, así como una exploración física diseñada para identificar las lesiones cardiovasculares que puedan provocar muerte súbita o progresión de la enfermedad ${ }^{22}$.

El principal problema que se plantea con los reconocimientos multitudinarios es la seguridad y reproducibilidad de la prueba utilizada. La seguridad se define por la sensibilidad y especificidad, de forma que se debe intentar minimizar el numero de individuos que, aun siendo de riesgo, pudieran quedar inadecuadamente tranquilizados (falsos negativos); como el de aquellos que pudieran ser innecesariamente apartados, temporal o definitivamente, de la práctica deportiva (falsos positivos) con el consiguiente costo económico y personal que una decisión errónea puede llevar asociada.

La exploración física meticulosa puede alertar sobre la presencia de ciertas cardiopatías, pero desgraciadamente la mayoría de los deportistas jóvenes con patologías de riesgo son asintomáticos, tienen una exploración física normal y presentan un excelente rendimiento deportivo.La inclusión del electrocardiograma de reposo aumenta la probabilidad de identificar a aquellos sujetos de riesgo, ya que es anormal en el $95 \%$ es personas con $\mathrm{MCH}$ y está alterado en la MCA, en los síndromes de QT largo y en el Wolfparkinson-white ${ }^{1223}$. Pero el electrocardiograma no tiene capacidad para detectar las anomalías de las arterias coronarias y éstas podrian descubrise por ecocardiografia ${ }^{24}$.

El modelo italiano (el de mayor experiencia en el uso extensivo de la ecocardiografía como método de estudio en deportistas) no tiene conclusiones definidas dado que en Italia no existe un registro nacional de los sujetos excluidos de la práctica deportiva por patologías de riesgo ni un registro nacional de muerte súbita.

Sin embargo, los resultados de la experiencia italiana permiten extraer algunas consideraciones sobre la eficacia del rastreo en la identificación de personas con riesgo. $\mathrm{La} \mathrm{MCH}$ aparece en una proporción baja entre los deportistas italianos fallecidos súbitamente en comparación con la estadísticas americanas. Tal discrepancia de resultados no se debe a la menor prevalencia de la enfermedad, sino seguramente a que a los deportistas italianos se los excluyó en los exámenes precompetitivos.

En cuanto a la MCA, el rastreo de la misma parece tener menor eficacia. La incidencia de esta patología es mayor en los deportistas fallecidos súbitamente que entre los no deportistas, hallazgo que se ratifica en los resultados de las series españolas ${ }^{14}$.Sin embargo la mayor parte de los deportistas fallecidos súbitamente presentaban anomalías relevantes en la anamnesis, examen clínico o electrocardiograma, pero el médico no fue capaz de acertar en el diagnóstico, probablemente porque ni siquiera llegó a sospechar o indagar la presencia de esta patología. En el futuro, una mayor información sobre las características de esta enfermedad miocárdica, hasta el momento poco conocida, podría mejorar la eficacia del reconocimiento.

Con respecto a las anomalías congénitas de las arterias coronarias, la valoración precompetitiva resulta desalentadora. La historia familiar en general es negativa, y rara vez presentan síntomas. Por otro lado, solo una minoría tiene anomalías en el electrocardiograma. El hallazgo en vida de estas anomalías es excepcional, por lo que parece razonable que el diagnóstico pueda escapar al médico examinador. La ergometría en estos pacientes suele ser normal o solo presentar arritmias ventriculares. Además, aquellas personas que practican disciplinas deportivas con moderada o baja exigencia física, podrían llegar a la alta competencia con estas anomalías.

El ecocardiograma en algunas ocasiones permite visualizar el ostium y el trayecto proximal de las arterias coronarias, cuando la ventana acústica es adecuada.

\section{Criterios para la toma de decisiones}

Cuando se identifican anormalidades cardiovasculares en deportistas jóvenes nos debemos preguntar: ¿Cuál es el riesgo de muerte si el atleta continúa haciendo deporte? ¿Cuánto se le reduce el riesgo si el atleta deja de hacer deporte? ¿Cuál criterio utilizo para aconsejar (o no aconsejar) que continúe haciendo deporte?

Los participantes de eventos deportivos con anormalidades cardiovasculares tienen un riesgo asociado a la actividad física intensa que no puede ser cuantificado con precisión considerando las condiciones extremas e imprevisibles a las cuales pueden ser expuestos. Además, en no todos los deportistas con $\mathrm{MCH}$ que mueren súbitamente, esta muerte se asocia a la actividad física intensa. Por otra parte, no todos los atletas con enfermedad cardiovascular mueren durante una competencia.

Aunque no se realizaron estudios formalmente controlados, las pruebas indirectas y la intuición clínica sugieren que la selección y estrategias de descalificación sean bien justificadas y probablemente reduzcan el número de muertes repentinas en atletas jóvenes.

Si tenemos en cuenta que, según las series publicadas, las causas 
de muerte súbita en los jóvenes deportistas son bastantes diferentes según el país que estemos analizando, realizar un ecocardiograma de rutina en EE.UU.podría detectar anomalías de la arterias coronarias.Pero en las mejores condiciones de técnica ecocardiográfica y ventana acustica, deberian realizarse 20 millones de ecocardiogramas para prevenir 13,5 muertes. De tal manera, lo que deberíamos hacer es tener registros serios de muerte súbita en esta población, conocer las causas de las mismas y en función de esto hacer un algoritmo de rastreo específico.

\section{Conclusiones}

Las consideraciones previas nos llevan a recomendar que a la hora de evaluar a personas menores de 35 años antes de la actividad deportiva recreacional, debemos realizarles una anamnesis profunda, examen físico y electrocardiograma. Si todo esto es normal la probabilidad de que tenga muerte súbita relacionada al deporte es muy baja.Si el paciente tiene historia familiar de muerte súbita, presenta un soplo o alteraciones electrocardiográficas deberíamos solicitar un ecocardiograma.

Si la personas a evaluar realiza actividad deportiva competitiva organizada en ligas o federaciones, nacionales o inter nacionales, se sugiere realizar un ecocardiograma de rutina, debido al riesgo que le agrega la competencia.

Dr. Federico Achilli [ Servicio de Cardiología.Hospital Italiano de Buenos Aires ]

\section{Referencias:}

1.Sudden cardiac death. Report of a WHO Scientific Group.World Health Organ Tech Rep Ser, 1985.726:p.5-25.

2.Siscovick, D.S., et al., The incidence of primary cardiac arrest during vigorous exercise.N Engl J Med, 1984.311(14):p.874-7.

3.Maron, B.J., L.C.Poliac, and W.O.Roberts, Risk for sudden cardiac death associated with marathon running.J Am Coll Cardiol, 1996.28(2):p.428-31

4.Maron, B.J., T.E.Gohman, and D. Aeppli, Prevalence of sudden cardiac death during competitive sports activities in Minnesota high school athletes. J Am Coll Cardiol, 1998.32(7): p. 1881-4.

5.Boraita Perez, A.and L.Serratosa Fernandez, [Sudden death (IV).Sudden death in the athlete. The minimal requirements before performing a competitive sport].Rev Esp Cardiol, 1999. 52(12):p.1139-45

6.Maron, B.J., et al., Sudden death in young competitive athletes.Clinical, demographic, and pathological profiles.Jama, 1996.276(3):p.199-204

7.Thiene, G., C.Basso, and D.Corrado, Is prevention of sudden death in young athletes feasible? Cardiologia, 1999.44(6):p.497-505.

8.de Vreede-Swagemakers, J.J., et al., Out-of-hospital cardiac arrest in the 1990's:a population-based study in the Maastricht area on incidence, characteristics and survival.J Am Coll 8.de Vreede-Swagemakers, J.

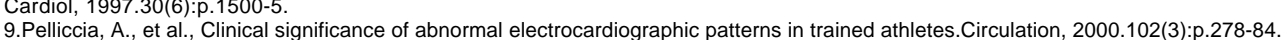

9.Pelliccia, A., et al., Clinical significance of abnormal electrocardiographic patterns in trained athletes.Circulatior
10.Futterman, L.G.and R.Myerburg, Sudden death in athletes:an update.Sports Med, 1998.26(5):p.335-50.

11.Marrugat, J., R.Elosua, and M.Gil, [Epidemiology of sudden cardiac death in Spain].Rev Esp Cardiol, 1999.52(9):p.717-25.

12.Maron, B.J., Sudden death in young athletes.N Engl J Med, 2003.349(11):p.1064-75.

13.Maron, B.J., et al., Relationship of race to sudden cardiac death in competitive athletes with hypertrophic cardiomyopathy.J Am Coll Cardiol, 2003.41(6):p.974-80

14.Suarez-Mier, M.P.and B.Aguilera, [Causes of sudden death during sports activities in Spain].Rev Esp Cardiol, 2002.55(4):p.347-58.

15.Priori, S.G., et al., Task Force on Sudden Cardiac Death, European Society of Cardiology.Europace, 2002.4(1):p.3-18.

16.Thiene, G., et al., Right ventricular cardiomyopathy and sudden death in young people.N Engl J Med, 1988.318(3):p.129-33.

17.Maron, B.J., et al., Clinical profile and spectrum of commotio cordis.Jama, 2002.287(9):p.1142-6

18.Link, M.S., et al., Upper and lower limits of vulnerability to sudden arrhythmic death with chest-wall impact (commotio cordis).J Am Coll Cardiol, 2003.41(1):p.99-104.

19.Boraita, A., [Sudden death and sport.Is there a feasible way to prevent it in athletes?].Rev Esp Cardiol, 2002.55(4):p.333-6.

20.Boraita Perez, A., [Dying for sport?].Med Clin (Barc), 2002.118(13):p.506-7.

21. Thiene, G., C.Basso, and D.Corrado, Sudden death in the young and in the athlete:causes, mechanisms and prevention.Cardiologia, 1999.44 Suppl 1(Pt 1):p.415-21.

22.American College of Sports Medicine Position Stand and American Heart Association.Recommendations for cardiovascular screening, staffing, and emergency policies at health/fitness facilities.Med Sci Sports Exerc, 1998.30(6):p.1009-18.

ness facilities.Med Sci Sports Exerc, 1998.30(6):p.1009-18.
23.Maron, B.J., Hypertrophic cardiomyopathy:a systematic review.Jama, 2002.287(10):p.1308-20.

23.Maron, B.J., Hypertrophic cardiomyopathy:a systematic review.Jama, 2002.287(10):p.1308-20.
24. Pelliccia, A., A.Spataro, and B.J. Maron, Prospective echocardiographic screening for coronary artery anomalies in 1,360 elite competitive athletes. Am J Cardiol, 1993.72(12): p. 978 9 .
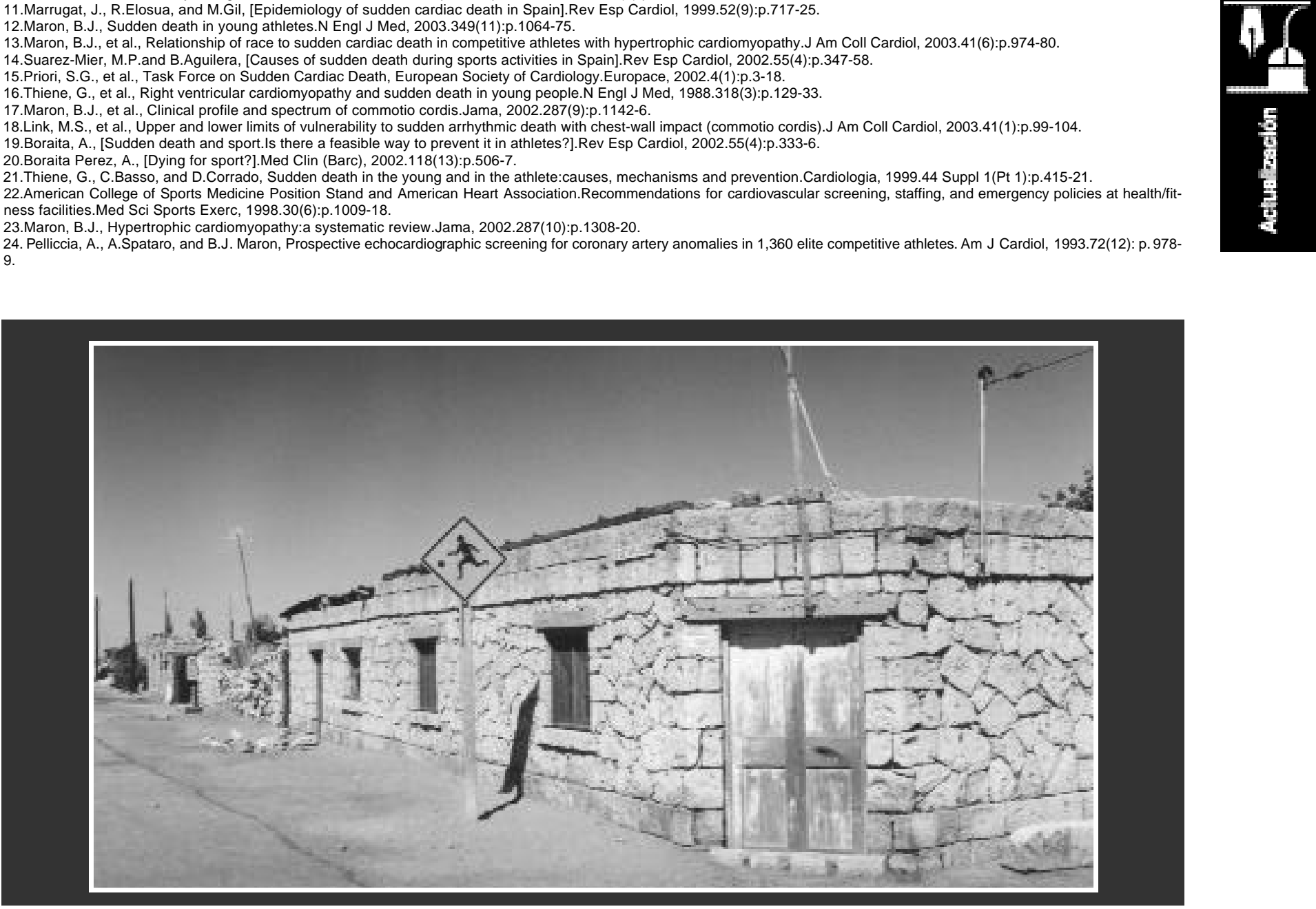